\title{
El debate entre ciencia y cristianismo en torno al origen del hombre en el pensamiento de Mariano Artigas
}

\section{The debate between science and Christianity about the origin of man in the thought of Mariano Artigas}

\author{
CARLOS ALBERTO MARMELADA \\ Universitat Internacional de Catalunya, de Institució Familiar d'Educació \\ carlosalbertomarmelada@yahoo
}

\begin{abstract}
Resumen. Entre las grandes inquietudes intelectuales del profesor Artigas estuvo siempre el aclarar las relaciones de la ciencia y la fe en aquellos campos en los que abordan cuestiones fronterizas, como es el caso de la naturaleza y origen del hombre. En este punto hay quienes han querido ver en la ciencia el fundamento intelectual de la increencia actual, la base legítima del ateísmo contemporáneo, que se sustentaría en la objetividad del conocimiento científico; especialmente la teoría de la evolución, que habría servido, desde los tiempos de Darwin, para demostrar que ya era legítimamente intelectual ser ateo; es decir, que la ciencia habría hecho innecesaria la hipótesis de Dios. Artigas dedicó numerosos y extensos trabajos a deconstruir este a priori cultural de nuestro tiempo, explicando cómo las nociones de evolución y creación no son antitéticas, y como la existencia del azar en la naturaleza no invalida la teleología a través de la acción de un plan divino; de modo que no hay contradicción en afirmar un origen biológico para el hombre con la existencia de facultades espirituales que le abren hacia la trascendencia. Denunciada la manipulación ideológica de la teoría de la evolución para fines espurios, Artigas aborda con la misma honestidad y valentía la compleja cuestión del monogenismo y el poligenismo. Nuestro breve artículo presenta un sucinto repaso sobre el desarrollo de estas cuestiones en su pensamiento.
\end{abstract}

Palabras clave: Ciencia; creación; Dios; darwinismo; finalidad; hombre; plan divino; teoría de la evolución.

Scientia $e t$ Fides $4(2) / 2016,79-95$ 
Resumen. One of the main intelectual concerns of Professor Artigas was to make clear the relationships between science and faith in bordering fields such as the nature and the origin of mankind. At this point, there are some researchers who have seen in science the intelectual basement of nowadays unbelief, the legitimous basement of modern atheism, which would find support in the objectivity of the scientific knowledge; especially the evolution theory, which would have been useful, from Darwin's times, to prove that it was legitimate intelectually speaking to be an atheist. I mean, science would have made useless the hypothesis of the existence of God. Artigas dedicated a lot of extensive essays to refute this modern affirmation, explaining how the terms of evolution and creation are not opposite, and how the existence of chance in nature doesn't deny the theology through the action of a divine plan, in a way that there's no contradiction in reassure a biological origin for mankind with the existence of espiritual faculties which lead to transcendency. Once reported the ideologic manipulation of the theory of evolution for spurious objectives, Artigas deals with the same honesty and braveness the complicated questions of monogenism and poligenism. Our brief article presents a concise review on the development of all these questions based on his thoughts.

Palabras clave: Science; creation; God; Darwinism; purpose; man; divine plan; theory of evolution.

\section{Amor por la sabiduría}

“Todos los hombres desean por naturaleza saber" (Aristóteles 1982, 4). Con esta llamativa afirmación el gran filósofo griego Aristóteles inicia su famosa obra titulada Metafísica. Sin duda alguna, se trata de unas palabras que, con toda justicia, se pueden aplicar perfectamente a Mariano Artigas; pues sus profundas inquietudes intelectuales le llevaron a doctorarse en filosofía, teología y física.

Este apetito natural por lograr una comprensión cabal de la totalidad de la realidad hizo que Artigas se sumergiera en una profunda y esmerada investigación de toda una serie de cuestiones relativas a una pluralidad de disciplinas tan variadas, pero intrínsecamente conectadas, como pueden ser la cosmología, la antropología, la filosofía de la naturaleza, la metafísica, la teología (tanto la natural como la revelada), la filosofía de la ciencia en general y la epistemología en particular, así como ciertos puntos cruciales de la historia de la ciencia (como podría ser, por ejemplo, el estudio pormenorizado del caso Galileo). Toda esta pluralidad de saberes teoréticos convergía 
en el espíritu de Artigas en un punto focal: conocer verdaderamente el fundamento último de la realidad, de tal manera que nuestra mente fuera capaz de elaborar un relato comprensivo e integral de la totalidad del ser, incluida la aprehensión de la verdadera esencia humana.

En este trabajo nos ocuparemos de uno de los temas centrales de este ambicioso y complejo proyecto intelectual de Artigas: el conocimiento del hombre, tanto en lo tocante a su origen y evolución biológica, como en lo relativo a su auténtica dimensión ontológica. En el horizonte espiritual de Artigas, aclarar estas cuestiones estuvo presente en su ánimo desde bien joven. Lograr conocer el verdadero lugar que ocupa el hombre en la naturaleza fue una inquietud que muy pronto despertó en su alma.

Decía el filósofo alemán Martin Heidegger que:

en ninguna época se ha sabido tanto y tan diverso con respecto al hombre como en la nuestra. En ninguna época se expone el conocimiento acerca del hombre en forma más penetrante y fascinante que en esta. Ninguna época, hasta la fecha, ha sido capaz de hacer accesible este saber con la rapidez y la facilidad que la nuestra. Y, sin embargo, en ninguna época ha sido el hombre tan problemático como en la actual. (Heidegger 1986, 177)

Heidegger apunta con su habitual agudeza que nunca antes se había sabido tanto acerca del hombre como ahora. En efecto, múltiples disciplinas como la psicología, la antropología, la sociología, la Historia, la arqueología, la paleontología o la biología, nos aportan un caleidoscopio de información y conocimiento acerca de nosotros que causaría un enorme asombro y perplejidad a cualquier sabio de hace una o dos centurias. Pero, al mismo tiempo, ese halo de misterio que ha envuelto al hombre, no solo permanece en pie, sino que se agiganta a medida que profundizamos en nuestro propio conocimiento. Otro filósofo alemán, Friedrich Wilhelm Nietzsche, reparó en esto mismo al afirmar que: "nosotros los que conocemos somos desconocidos para nosotros" (Nietzsche 1987, 17). Unas palabras que invitan a una sugerente reflexión, y que en el caso de Heidegger le conminaron a ver a la humanidad como unos “apátridas en la patria más propia” (Heidegger 1989, 130). Explicitar cuál es esa patria y en qué consiste el auténtico estatuto 
ontológico del hombre en dicha patria es a lo que consagró Artigas todo su esfuerzo intelectual en el campo de la antropología, tanto en su vertiente filosófico-teológica como científica.

\section{2.¿Qué es el hombre?}

Hacia el final de la Crítica de la razón pura Kant sostiene que las tres cuestiones más importantes que se pueden plantear los seres humanos son: ¿Qué puedo saber? ¿Qué debo hacer? y ¿Qué puedo esperar? El primer interrogante hace referencia a la naturaleza del conocimiento humano, a la comprensión de su origen, sus límites, su fiabilidad y alcance. El segundo se refiere a la conducta moral consubstancial al hecho de ser humanos y el tercero a la inmortalidad del alma y la existencia de Dios. Lo sorprendente es que el filósofo prusiano reduce estas tres preguntas a una más básica: ¿qué es el hombre? Convirtiéndola así en la más importante de todas. No es que Kant reduzca la epistemología (o la gnoseología en general), la ética y la teología natural a antropología, sino que considera que sabiendo bien qué es el hombre podremos enfocar adecuadamente todas las otras cuestiones.

Tal vez la pregunta ¿qué es el hombre? no sea la más importante de todas (algunos dirán que es la relativa a si Dios existe verdaderamente de un modo objetivo o no), lo que sí es indiscutible es que se trata de una de las más fundamentales. También está fuera de toda duda que la cuestión del hombre está ligada a la de Dios, pues tanto nuestra identidad (¿quiénes somos?), como nuestro origen (¿de dónde venimos?) y nuestro destino (¿a dónde vamos?, que es el equivalente popular al kantiano ¿qué puedo esperar?) están totalmente comprometidos en la cuestión de la existencia o no de Dios. Artigas ha planteado este asunto de forma explícita al preguntarse si somos unos seres puramente materiales que existimos gracias al azar, de tal suerte que todo se acaba para nosotros con la muerte; o si, por el contrario, contamos con una dimensión espiritual creada por Dios que nos abre las puertas a la inmortalidad y que confiere un sentido trascendente a nuestra existencia (Artigas y Turbón 2008, 19). 


\section{Emergentismo: más a partir de menos}

En el primer caso los materialistas han de explicar cuál es el origen de las llamadas facultades espirituales que tiene el hombre: la inteligencia y la voluntad (con su capacidad única de amar libremente). Según ellos estas habrían surgido gradualmente a lo largo del proceso evolutivo, una postura que Artigas califica de "emergentismo"1. Para él los elementos espirituales que caracterizan a los humanos ("más") no pueden proceder de la potencialidad que encierra la materia ("menos"), sino que suponen un salto ontológico; de modo que la diferencia entre el hombre y el resto de los vivientes es cualitativa y no meramente de grado, como sostendría el materialismo emergentista. Es cierto que el hombre es un ser que cuenta con una base biológica de la misma naturaleza que la de los otros vivientes, y que esta dimensión biológica parece ser dinámica (evolucionismo), pero Artigas insiste en que esto no anula el hecho de que el hombre encierre una novedad esencial respecto a todos los otros entes naturales vivos; o lo que es lo mismo: el hombre es un animal, pero no es solo un animal.

Para los emergentistas las cualidades humanas fueron surgiendo gradualmente en los homínidos prehumanos (quizás alguna especie de australopitecino como pudiera ser A. africanus, A. garhi o alguna otra aún no descubierta, incluso algún otro género de homínido no hallado todavía). Para los que sostienen que Dios tiene una relación directa con ese salto ontológico que da lugar al hombre la pregunta es cuándo y en quién se produjo. Para Artigas no es posible responder científicamente a estas cuestiones. Según él las “dimensiones espirituales comenzaron a existir en el ser humano en algún momento, cuando existía la base biológica necesaria. No sabemos cuándo fue y posiblemente no lo sabremos nunca” (Artigas y Turbón 2008, 127).

La respuesta a la pregunta por el cuándo está relacionada con el quién. Dicho de otro modo: ¿A qué especie de homínido pertenecieron Adán y Eva? ¿Los primeros padres de la humanidad fueron la primera pareja de Homo sapiens? ¿O tal vez la primera pareja de Homo habilis, o de Homo rudolfensis?

Para un análisis de lo que entiende Artigas por materialismo emergentista, ver Artigas 1992a, 483-489. 
En el primer caso la humanidad tendría una antigüedad que rondaría los 200.000 años. En el segundo estaríamos hablando de unos dos millones y medio de años. Fiorenzo Facchini sostiene que este tema lo han de dilucidar los científicos y no los filósofos o los teólogos (Artigas y Turbón 2008, 195).

\section{Monogenismo y poligenismo}

Una cuestión mucho más problemática desde el punto de vista doctrinal es si el Adán y la Eva bíblicos ${ }^{2}$ representan a una pareja de individuos reales de la que procedería toda la humanidad; o si se trata de un símbolo que hace referencia a una primera población formada por varios protohumanos que habrían aparecido simultáneamente a partir de una especie de homínido prehumana. Es decir: ¿el origen de la humanidad es monogénico o poligénico?

A nadie se le escapa que esta cuestión no es, en absoluto, baladí, pues tiene que ver con la doctrina judeocristiana del pecado original. Artigas, en su citado libro sobre el origen del hombre publicado junto al catedrático de biología Daniel Turbón, después de exponer las ventajas biológicas que ofrece el monogenismo (facilitar la reestructuración del genotipo para presentar novedades determinantes en el surgimiento de una nueva especie) llama la atención sobre un hecho de gran interés, y es que "la actual edición del Catecismo de la Iglesia Católica no menciona el término monogenismo" (Artigas y Turbón 2008, 147). En otro lugar, después de señalar que: “por grande que sea el progreso científico, parece muy difícil llegar a conclusiones claras acerca del monogenismo o el poligenismo contando solo con la ciencia [...]. Por otra parte, aunque el monogenismo plantee algunas dificultades a nuestro afán de representar el origen de la especie humana, el poligenismo también plantea dificultades nada triviales" (Artigas 2007, 165). Artigas advierte que, aunque "existen posibilidades científicamente respetables para explicar el origen monogenista del hombre actual [...] el poligenismo no ha sido excluido de un modo absoluto" (Artigas 2007, 166),

2 No confundir con la Eva africana o mitocondrial y con el Adán ZFY; que, además, no fueron coetáneos entre sí. 
de tal manera que algunos acaban observando que ciertos "teólogos han intentado mostrar que podría existir esa conciliación, aunque se trata de un tema que presenta dificultades" (Artigas 2007, 166). Lo cierto es que no son pocas las dificultades a las que alude Artigas; pues, tal como señala Pio XII: "No se ve cómo tal opinión [se refiere a la poligenista] pueda compaginarse con lo que las fuentes de la verdad revelada y las enseñanzas del Magisterio de la Iglesia proponen acerca del pecado original” (Pío XII 1950, 576).

\section{El Vaticano y la recepción del darwinismo}

La delicadísima y compleja cuestión del monogenismo y el poligenismo, nos lleva a tratar otro de los temas a los que Artigas dedicó gran atención en sus últimos años: la actitud de la Congregación del Índice frente a los autores católicos que defendían la compatibilidad entre la teoría científica de la evolución biológica y la doctrina cristiana. Es decir, se trataba de analizar cuál había sido la postura oficial del Vaticano respecto a dicha teoría desde que esta se dio a conocer en la segunda mitad del siglo XIX; y, de paso, ver la posición de los papas en relación con la evolución del hombre. Esto fue posible a partir de la apertura del Archivo de la Congregación para la Doctrina de la Fe en 1998. Investigar este asunto le llevó al año siguiente hasta Roma, con la finalidad de estudiar los archivos vaticanos (para un análisis detenido de esta cuestión ver Martínez 2007, 529-249).

Revisando documentos en busca de la recepción del darwinismo por parte de la Santa Sede, Artigas se llevó la inesperada y fascinante sorpresa de toparse con el hallazgo de un manuscrito inédito en el que se juzgaba la doctrina atomista de Galileo en su popular obra Il Saggiatore. No es necesario remarcar el enorme valor histórico de un material de este calibre. La traducción y el estudio en cuestión llevaron un par de años. Una vez concluida esta tarea, que se vio culminada con la publicación del manuscrito (Artigas et al. 2001, 197) y de un trabajo sobre el contenido y sus implicaciones (Artigas, Martínez y Shea 2003, 159), Artigas retomó el proyecto original, al que se había incorporado Rafael A. Martínez. 
El enorme progreso de las ciencias empiriométricas de la naturaleza producido desde su aparición en el siglo XVII, desembocó en el siglo XIX en una confrontación entre ciencia y cristianismo. A ciertos intelectuales de la época les interesó, por cuestiones puramente ideológicas, presentar a ambos como enemigos inevitables e irreconciliables.

La tensión entre ciencia y religión se acentuó en la segunda mitad del siglo XIX a raíz de la publicación y divulgación de las ideas darwinianas sobre la evolución de las especies, teniendo a la selección natural como mecanismo explicativo del cambio o transformación. En esta época la teología se veía atacada constantemente por aquellos que usaban a la ciencia como un arma capaz de desprestigiar a la religión. De este modo, para algunos la teoría de la evolución dejaba en evidencia al milenario relato bíblico de la creación del hombre por parte de Dios, ofreciendo una alternativa naturalista que hacía las delicias de los monistas materialistas. En este contexto, no es de extrañar que hubiera teólogos que vieran con recelo la teoría de la evolución y apostaran por su denuncia; pero también hubo teólogos cristianos que estimaron plausible la elaboración de una síntesis que apostara por compatibilizar dicha teoría con la concepción antropogénica incluida en el relato del Génesis.

Los estudios de Artigas llevados a cabo en relación con los Archivos del Índice arrojaron como conclusión que las autoridades vaticanas nunca pronunciaran una condena oficial contra la teoría científica de la evolución biológica, aunque sí hubo amonestaciones contra sus partidarios. Es decir, no existió una política oficial del Vaticano en contra del evolucionismo como tal (entendiéndolo aquí como teoría científica y no como movimiento ideológico), ni un patrón común en la toma de decisiones de papas y cardenales, sino que se obró atendiendo a las circunstancias específicas de cada momento.

Artigas y sus colaboradores analizaron seis casos concretos. En los de Bonomelli, Hedley y Mivart "no existió ninguna acción contra estos autores" (Martínez 2007, 548). En los tres casos en los que intervino la Congregación del Índice (los de Caverni, Leroy y Zahm) “lo hizo respondiendo a denuncias externas. El Santo Oficio no intervino en ninguno de los casos. Puede afirmarse que los casos examinados no respondieron a una política de 
las autoridades romanas contra el evolucionismo" (Martínez 2007, 548). En resumidas cuentas: "la evolución, no ha sido nunca objeto de ninguna condena oficial por parte de las autoridades vaticanas" (Artigas 2007, 9).

Para nuestro autor, la causa de la ausencia de una condena oficial estuvo en la voluntad, por parte del Vaticano, de evitar la repetición de un nuevo caso Galileo. En efecto:

Las autoridades del Vaticano eran conscientes de que no existía una decisión doctrinal acerca del evolucionismo, y según parece no tenían excesivo interés en provocarla. Examinaron los diversos escritos en respuesta a denuncias concretas, e intentaron analizarlos en base a la doctrina existente, sin seguir ninguna directiva explícita acerca de la cuestión. Eso explica que los diversos informes, muy distintos en cuanto a longitud, argumentos y conclusiones, no siguieran ningún esquema uniforme [...]. Es muy probable que a la suavidad de las medidas adoptadas contribuyera el deseo de no comprometer la autoridad de la Iglesia en un terreno relacionado con la ciencia. Las autoridades romanas no querían hallarse ante un nuevo “caso Galileo”. (Martínez 2007, 549)

En resumen: "El Magisterio de la Iglesia nunca ha condenado las teorías científicas de la evolución, y admite que estas teorías se pueden compaginar con el cristianismo, siempre que se respeten los aspectos básicos de la doctrina católica acerca de la acción de Dios y la persona humana” (Artigas y Turbón 2008, 147). Y es que "la Iglesia Católica no se ha pronunciado nunca, de modo oficial, contra las teorías evolucionistas, con tal que estas no se extrapolen fuera del campo científico" (Artigas 1992b, 98).

\section{Los papas y el evolucionismo}

El estudio de la investigación llevada a cabo por Artigas con respecto a la recepción del darwinismo por parte de las autoridades vaticanas puede complementarse con la exposición que hace de la opinión de diversos papas sobre la teoría de la evolución.

En 1950 el papa Pío XII publicó la encíclica Humani generis, en ella se incluye un párrafo que ha servido durante décadas como punto de referencia 
para sostener la compatibilidad entre cristianismo y evolucionismo. El texto en cuestión es el siguiente:

El magisterio de la Iglesia no prohíbe que, según el estado actual de las ciencias humanas y de la Sagrada Teología, se trate en las investigaciones y disputas de los entendidos en uno y otro campo, de la doctrina del "evolucionismo", en cuanto busca el origen del cuerpo humano en una materia viva preexistente - pues las almas nos manda la fe católica sostener que son creadas inmediatamente por Dios-; pero de manera que con la debida gravedad, moderación y templanza se sopesen y examinen las razones de una y otra opinión, es decir, de los que admiten y los que niegan la evolución. (Pío XII 1950)

En abril de 1988 la universidad de Múnich organizó en Roma un Simposio internacional sobre La fe cristiana y la teoría de la evolución, al que asistió el por entonces cardenal Ratzinger y que dirigieron Robert Speamann y Reinhard Low, quien afirmó que "una teoría de la evolución bien formada no solo puede ser aceptable, sino perfectamente compatible con la fe" (Artigas 1992b, 97); hasta el punto de que "la teoría de la evolución, si se mantiene en sus justos límites, no solo no choca la fe, sino que, de alguna manera, resalta su esplendor" (Artigas 1992b, 97). El propio papa Juan Pablo II afirmó allí que "el debate en torno al modelo explicativo de evolución no encuentra obstáculo en la fe, con tal que la discusión permanezca en el contexto del método naturalista y de sus posibilidades" (Artigas 1992b, 97). Al año siguiente, y durante una Audiencia General, Juan Pablo II recoge las citadas palabras de Pío XII y las explicita señalando que "es posible, según la hipótesis mencionada, que el cuerpo humano, siguiendo el orden impreso por el Creador en las energías de la materia, haya sido preparado gradualmente en las formas de seres vivientes antecedentes” (Juan Pablo II 1981, 1041). Una década más tarde hizo unas declaraciones especialmente relevantes al señalar que:

nuevos conocimientos llevan a pensar que la teoría de la evolución es más que una hipótesis. En efecto, es notable que esta teoría se haya impuesto paulatinamente al espíritu de los investigadores, a causa de una serie de descubrimientos hechos en diversas disciplinas del saber. La convergencia, de ningún modo 
buscada o provocada, de los resultados de trabajos realizados independientemente unos de otros, constituye de suyo un argumento significativo a favor de esta teoría. (Juan Pablo 1996, 4)

Esto no significa la aceptación acrítica de cualquier propuesta evolucionista, sino de aquellas que no trascienden los límites de la ciencia positiva y que no realizan afirmaciones, más bien de carácter filosófico, que se pronuncian con un claro tono ideológico al hacerlas pasar por conclusiones científicas.

Benedicto XVI, siendo el cardenal Ratzinger, ya había tocado el tema en su obra Creación y pecado en donde afirma que:

no podemos decir: creación o evolución; la manera correcta de plantear el problema debe ser: creación y evolución, pues ambas cosas responden a preguntas distintas. La historia del barro y del aliento de Dios [...] no nos cuenta cómo se origina el hombre [...]. Y a la inversa, la teoría de la evolución trata de conocer y describir periodos biológicos. Pero con ello no puede aclarar el origen del "proyecto" hombre, su origen íntimo ni su propia esencia. Nos encontramos, pues, ante dos preguntas que en la misma medida se complementan y que no se excluyen mutuamente. (Ratzinger 2005, 75)

Ratzinger consideró tan importante la cuestión del origen del hombre que aludió a ello en su homilía inicial de papado al afirmar allí que: "no somos el producto casual y sin sentido de la evolución. Cada uno de nosotros es querido, cada uno es amado, cada uno es necesario" (Benedicto XVI 2005). Las dos cuestiones traídas a colación por estos textos de Ratzinger ponen encima de la mesa el tema de la compatibilidad entre las nociones de creación y evolución y la de una finalidad en la naturaleza; de tal modo que Dios tiene un plan, un proyecto, para el hombre, no siendo la existencia de este el mero fruto del azar. Veamos ambas cuestiones.

\section{Compatibilidad entre evolución y creación}

Para Artigas "las presuntas oposiciones entre evolución y acción divina carecen de base” (Artigas y Turbón 2008, 90). Y es que "la evolución no lleva, por sí sola, a afirmar ni a negar la acción de Dios en el mundo. Los científicos 
estudian la evolución sin contar con Dios, porque buscan explicaciones naturales, pero eso no quiere decir que nieguen a Dios. Significa, simplemente, que la biología se limita a lo que se puede conocer mediante los métodos de las ciencias” (Artigas y Turbón 2008, 87). Por tanto, se puede afirmar “que no existe la alternativa 'evolución-creación', como si se tratara de dos alternativas entre las que haya que elegir. Se puede admitir la evolución y, al mismo tiempo, la creación divina” (Artigas 1992c, 152). De hecho, “Dios pudo crear el universo en estados muy diversos, y esto nada choca con la posibilidad de que posteriormente unos seres surgieran a partir de otros" (Artigas 1992c, 153). Para Artigas la conclusión es evidente: "Las teorías de la evolución nada tienen que objetar a la necesidad de admitir un Creador. Esas teorías solo estudian el origen de unos vivientes a partir de otros, pero siempre quedará por responder cuál es la causa última de la existencia de todo lo que existe, y a ese nivel es necesario admitir la existencia de un Dios creador" (Artigas 1992c, 155). De modo que:

según las enseñanzas de la iglesia católica, no existe oposición entre la doctrina católica y las teorías evolucionistas, con tal de que estas se valoren con el necesario rigor, lo cual supone, entre otras cosas, que no se utilicen fuera de su contexto científico tal como sucede cuando se realizan saltos injustificados que llevan a posiciones materialistas o a la negación y relativización de las verdades religiosas. Sin embargo, no son pocos los autores que dan ese salto injustificado hasta el materialismo, presentándolo como si estuviera justificado por la ciencia. (Artigas 1992c, 202)

En esta idea insiste en varios lugares; así en El hombre a la luz de la ciencia nos señala que "según las enseñanzas de la Iglesia católica, no existe oposición entre la doctrina católica y las teorías evolucionistas, con tal de que estas se valoren con el necesario rigor, lo cual supone, entre otras cosas, que no se utilicen fuera del contexto científico" (Artigas 1992b, 98).

Llegados a este punto Artigas se hace la pregunta clave: “ ¿Se puede ser, a la vez, evolucionista y cristiano?” (Artigas y Turbón 2008, 135). Su respuesta es clara: "Hoy día, los teólogos católicos dicen que sí, pues creación y evolución son compatibles; esta última no es sino la expresión dinámica de la 
primera" (Artigas y Turbón 2008, 135). De hecho, Artigas remarca que no solo son compatibles las nociones de evolución y creación, sino que aquella exige a esta; en el sentido de que para que algo pueda evolucionar antes ha tenido que ser creado, en el sentido de que, en primer lugar, la evolución se da dentro de la creación y, en segundo lugar, la totalidad de los entes contingentes (y el proceso evolutivo lo es) exige, en último término, un fundamento necesario y trascendente que los crea en un acto libérrimo y gratuito.

Así, pues, "la causalidad creada es compatible con la acción divina" (Artigas y Turbón 2008, 95). Por consiguiente: "si se entiende qué es la creación y la conservación en el ser, es fácil comprender que la acción de Dios no se sitúa en el plano de las causas creadas, y que debe afirmarse tanto si se admite la evolución como si no se admite” (Artigas 1992c, 195).

\section{Teleología}

Decíamos antes que la compatibilidad entre creación y evolución está vinculada con la idea de que existe una finalidad en la naturaleza (teleología) que se corresponde a un plan divino. Esta es, justamente, la gran cuestión. Así lo reconoce Artigas cuando advierte que:

el gran problema, en definitiva, es si somos objeto de un plan divino o hemos aparecido en la Tierra como simple resultado de leyes ciegas y del azar. Pero no se trata de extremos concluyentes. Para Dios, que es la Causa Primera que da el ser a todo lo que existe, y por tanto conoce todo perfectamente, no hay dificultad en que sus planes se realicen contando con leyes naturales de las que Él mismo es autor, y con la intervención de los que para nosotros es azar porque no podemos predecirlo. (Artigas 2007, 28)

El azar existe para nosotros en cuanto que es el desconocimiento de las causas, en cambio "para Dios no existe el azar, porque todo está sometido a su poder y conoce perfectamente todos los procesos y sus efectos" (Artigas 2007, 42). La conclusión que extrae Artigas es que: "no veo motivo para negar la evolución ni tampoco para minusvalorar el papel del azar y de la selección natural. Me parece que son aspectos que deben tener en cuenta 
una reflexión filosófica rigurosa en la actualidad. Sin embargo, también me parece que ello no autoriza a prescindir de la finalidad en el estudio de la naturaleza” (Artigas 2007, 74). Según Artigas, "no debería haber ningún problema para combinar la evolución y la existencia de un plan divino" (Artigas y Turbón 2008, 102), y esto es así porque "el mismo efecto puede ser considerado como contingente cuando se compara con sus causas inmediatas $\mathrm{y}$, al mismo tiempo, estar incluido dentro de un plan divino que no puede fallar" (Artigas y Turbón 2008, 103). La biología y la filosofía (especialmente la metafísica) abordan distintos planos ontológicos y epistemológicos de la realidad, no mediante una yuxtaposición, sino complementándose, por lo que "la combinación de azar y finalidad, de variación y selección, junto con las potencialidades para la autoorganización, pueden ser completadas fácilmente como el camino utilizado por Dios para producir el proceso de la evolución” (Artigas y Turbón 2008, 106).

En relación con este tema Artigas concluye que:

todo tiene su causa, pero muchas cosas suceden cuando se juntan causas independientes. A eso se llama azar: a la concurrencia de líneas causales independientes. El azar existe. Pero solo existe para nosotros. Para Dios, que es la Causa Primera de la que depende siempre todo, no hay azar ni casualidad. Por tanto, de la existencia del azar en la evolución no se puede concluir que no exista un plan divino y que el ser humano no sea el resultado previsto de ese plan. (Artigas y Turbón 2008, 107)

Negar la existencia de la finalidad en la naturaleza en nombre de la ciencia es forzarla a decir más de lo que sus métodos permiten afirmar, así, pues,

cuando se afirma que la combinación de la necesidad y del azar hace superfluo el recurso a una causa metafísica, se traspasan los límites de la perspectiva científica. Para afirmar la existencia de un plan divino, es necesario dar un salto metafísico cuya legitimidad no puede ser justificada mediante las ciencias. Pero, por el mismo motivo, las ciencias tampoco pueden mostrar que ese salto sea ilegítimo. (Artigas 1992a, 399)

Este detalle es muy importante, ya que: “la combinación de la necesidad y del azar es real. Puede ser suficiente para explicar parcialmente la naturaleza, 
mostrando qué tipos de procesos intervienen en el funcionamiento de la naturaleza, y cómo pueden surgir unas entidades a partir de otras. Pero no puede explicar el fundamento radical de la naturaleza” (Artigas 1992a, 399).

\section{La armonía entre razón y fe}

En resumidas cuentas, Artigas apuesta, argumentando con detalle, por la compatibilidad entre la visión evolutiva de la naturaleza viviente con la noción metafísica de la creación a partir de la nada, un hecho este que escapa a los métodos de investigación que usa la ciencia. En efecto, la ciencia empiriométrica de la naturaleza estudia las transformaciones producidas desde un estado físico concreto inicial a otro estado físico concreto final. La creación ex nihilo, en cambio, consiste en un paso desde la no existencia absoluta (y por lo tanto carente de toda caracterización física) a un estado físico, de modo que es un hecho que no puede ser objeto de estudio por parte de la ciencia.

Por lo que al ser humano se refiere, Artigas no ve incompatibilidad alguna entre el estudio de su desarrollo biológico evolutivo y la afirmación de que sus dimensiones espirituales son objeto de creación directa por parte de Dios. La ya mencionada compatibilidad entre la teoría de la evolución y la doctrina cristiana de la creación del hombre, por lo que a sus inquietudes intelectuales se refiere, se enmarca en el contexto de la compatibilidad general entre las verdades de fe y las verdades de razón; es decir, Artigas apuesta por la armonía entre ciencia, razón y fe. Por otra parte, hay quienes utilizan a la teoría de la evolución para intentar probar científicamente que Dios es una entidad ficticia y que la religión, por consiguiente, es un discurso supraestructural carente de base real.

\section{La manipulación ideológica de la teoría de la evolución}

Artigas ha denunciado por activa y por pasiva el uso manipulativo de cualquier teoría científica para intentar difundir ideología haciéndola pasar por ciencia. La teoría de la evolución ha sido, precisamente, una de las que más se ha esgrimido en contra de la religión ya que "el darwinismo suele ser 
utilizado en este contexto para afirmar que Darwin ha hecho posible ser ateo de modo intelectualmente legítimo, porque el darwinismo mostraría que no es necesario admitir la acción divina para explicar el orden que existe en el mundo" (Artigas 2007, 92). Este uso ideológico de la teoría de la evolución ha sido rechazado repetidamente por Artigas como algo que nada tiene que ver con la ciencia sensu estricto (Artigas 1992b, 99). De modo que reivindica una búsqueda sincera de la verdad, dejando al margen todos los prejuicios ideológicos. De hecho, hay una manifiesta contradicción en aquellos que esgrimen la ciencia en general (y la teoría de la evolución en particular) como una prueba de la verdad del materialismo, cuando este es, en realidad, una ideología filosófica y no una teoría científica empíricamente contrastada. Con profunda agudeza, Artigas resalta el hecho de que la misma ciencia que se utiliza para querer probar la verdad del materialismo y pretender demostrar científicamente que el hombre no es más que un animal, pues todo lo que existe sería puramente material (Artigas 1992b, 115; 2007, 21), es, precisamente, un ejemplo de la diferencia esencial entre el hombre y los animales (Artigas 2007, 26 y 31).

\section{Ese misterio llamado hombre}

La teoría científica de la evolución no se opone de suyo a la doctrina metafísica y teológica de la creatio ex nihilo, sino al fijismo (el convencimiento de que Dios creó a las especies tal como las conocemos hoy). Dios crea de forma gratuita; no necesita crear, ya que es perfecto, de modo que no se beneficia en nada de su creación, pues es imperfectible. ¿Entonces por qué crea? Lo hace para comunicar sus perfecciones y bondades a las creaturas (Artigas 2007, 26 y 36). En el caso del hombre Dios le hace partícipe de su vida espiritual y le ofrece la posibilidad de poder disfrutar eternamente de su gloria, único modo de colmar el ansia de plena felicidad al que todo hombre aspira por naturaleza. Ahora bien, haciendo uso de su libre albedrío el hombre puede aceptar o rechazar ese destino.

La de Artigas ha sido prácticamente una vida entera dedicada al estudio y a la búsqueda de la sabiduría que nos permita conocer verdaderamente 
cómo es el cosmos, el hombre y Dios. Una búsqueda que le llevó a concluir (en virtud de esas capacidades espirituales que especifican al hombre: intelectualidad, libertad y capacidad de amar buscando solo el bien del otro) que "cada humano es un misterio" en virtud de la riqueza prácticamente inagotable que encierra la interioridad humana (Artigas y Turbón 2009, 129).

\section{Referencias}

Aristóteles. 1982. Metafísica. Madrid: Gredos.

Artigas, Mariano. 1992a. La inteligibilidad de la Naturaleza. Pamplona: Eunsa.

-. 1992b. El hombre a la luz de la ciencia. Madrid: Palabra.

-. 1992c. Las fronteras del evolucionismo. Madrid: Palabra.

-. 2007. Ciencia y religión. Conceptos fundamentales. Pamplona: Eunsa.

Artigas, M., R. Martínez, F. L. Mateo-Seco, \& W. R. Shea. 2001. "Un inedito sul caso Galilei." Acta Philosophica 10:197-272.

Artigas, M., R. Martínez, y W. R. Shea. 2003. "Nueva luz en el caso Galileo.” Anuario de Historia de la Iglesia 12:159-179.

Artigas, M., y D. Turbón. 2008. Origen del hombre. Ciencia, filosofía y religión. Pamplona: Eunsa.

Benedicto XVI. 2005. Homilía del 24 de abril. Disponible en: https://w2.vatican.va/ content/benedict-xvi/es/homilies/2005/documents/hf_ben-xvi_hom_20050424_ inizio-pontificato.html

Heidegger, M. 1986. Kant y el problema de la Metafísica. México: FCE.

-. 1989. Conceptos fundamentales. Madrid: Alianza Editorial.

Juan Pablo II. 1986. Audiencia general: El hombre, imagen de Dios, un ser espiritual y corporal. 16 de abril. Insegnamenti IX, 1: 1041. Disponible en https://w2.vatican.va/ content/john-paul-ii/es/audiences/1986/documents/hf_jp-ii_aud_19860416.html -. 1996. "Mensaje a la Academia Pontificia de Ciencias.", 22 de octubre. Disponible en http://w2.vatican.va/content/john-paul-ii/es/messages/pont_messages/1996/ documents/hf_jp-ii_mes_19961022_evoluzione.html

Martínez, R. 2007. "El Vaticano y la evolución. La recepción del darwinismo en el Archivo del Índice.” Scripta Theologica 39:529-549.

Nietzsche, W. F. 1987. La genealogía de la moral. Madrid: Alianza Editorial.

Pío XII. 1950. Carta encíclica Humani generis. Disponible en http://w2.vatican.va/ content/pius-xii/es/encyclicals/documents/hf_p-xii_enc_12081950_humani-generis.html

Ratzinger, J. 2005. Creación y pecado. Pamplona: Eunsa. 